\title{
HITTING TIME BOUNDS FOR BROWNIAN MOTION ON A FRACTAL
}

\author{
WILLIAM B. KREBS
}

(Communicated by Lawrence F. Gray)

\begin{abstract}
We calculate a bound on hitting times for Brownian motion defined on any nested fractal. We apply this bound to show that any such process is point recurrent. We then show that any diffusion on a nested fractal must have a transition density with respect to Hausdorff measure on the underlying fractal. We also prove that any Brownian motion on a nested fractal has a jointly continuous local time with a simple modulus of space-time continuity.
\end{abstract}

\section{INTRODUCTION}

Recently the study of diffusion processes has been extended to inherently "rough" or nondifferentiable state spaces. Several authors have constructed diffusions on fractals, particularly, on the so-called "nested fractals." Diffusions have been constructed on the Sierpinski gasket by Goldstein [5], Kusuoka [8], and Barlow and Perkins [2], and on the Vicsek snowflake by Krebs [7].

In his 1990 monograph, Lindstrøm observed that nested fractals share common properties that permit a general construction of a diffusion on any fractal set satisfying a set of four axioms. (See Lindstrøm [9] for further details.) This raises the natural question of what properties are common to every diffusion on any nested fractal.

The fundamental fact about nested fractals is that they can be approximated to an arbitrary degree of accuracy by regular graphs. This makes it possible to approximate a diffusion on a nested fractal by a random walk on a reasonably simple graph. Properties of the diffusion can then be deduced from the properties of the approximating random walks.

In this work, we use random walks on the graphs approximating a nested fractal to estimate moments of the hitting times of the diffusion on the nested fractal. This gives us an estimate of the modulus of continuity of the diffusion. We then apply this simple bound to answer questions about the behavior of the diffusion. In particular, we can show that any diffusion on a nested fractal is point recurrent, that any such diffusion has a jointly continuous local time with a space-time modulus of continuity that we can estimate, and that any

Received by the editors August 28, 1990 and, in revised form, April 15, 1991.

1991 Mathematics Subject Classification. Primary 60J60.

Key words and phrases. Diffusions, fractals. 
such diffusion has a transition density with respect to Hausdorff measure on the underlying fractal.

A brief overview of the terminology of diffusions on nested fractals is given in $\S 1$. In $\S 2$, we estimate the mean of time required for the diffusion to hit an arbitrary point, starting from an arbitrary point. In $\S 3$, we apply the bound to solve several problems posed by Lindstrøm in [9].

\section{BROWNIAN MOTION ON NESTED FRACTALS}

In this section we present a brief review of nested fractals, as well as the theorems establishing the existence of Brownian motion on such sets. This exposition follows the comprehensive treatment of these ideas given in [9, Chapters I-V], which also provides some examples to motivate the ideas.

Let $\left\{\phi_{1}, \ldots, \phi_{\mu}\right\}$ be a set of $\nu$-similitudes in $\mathbf{R}^{n} \cdot \phi_{j}(x)=\nu^{-1} \cdot U_{j} x+b_{j}$ where $U_{j}: \mathbf{R}^{n} \rightarrow \mathbf{R}^{n}$ is an orthogonal transformation and $b_{j}$ is a fixed vector. For bounded subsets $A$ of $\mathbf{R}^{n}$, define $\mathscr{M}(A)=\bigcup_{1}^{\mu} \phi_{i}(A)$. It is well known that there exists a unique compact set $\Gamma$ such that $\Gamma=\mathscr{M}(\Gamma)$. (See, e.g., Hutchinson [6].)

It is well known that if $\phi_{j}$ is a $\nu$-similitude with $\nu>1$ then $\phi_{j}$ has a unique fixed point $x_{j}$ in $\mathbf{R}^{n}$. Let $F^{\circ}$ be the set of fixed points of $\left\{\phi_{1}, \ldots, \phi_{\mu}\right\}$. Say that a fixed point $x$ is essential if there exist $i \neq j$ such that $\phi_{i}(x)=\phi_{j}(x)$. Let $F$ denote the set of essential fixed points of the system $\left\{\phi_{1}, \ldots, \phi_{\mu}\right\}$. It is not hard to see that $F \subset \Gamma$.

For any $A \subset \mathbf{R}^{n}$, let $A^{(0)}=A$ and let $A^{(n)}=\mathscr{M}\left(A^{(n-1)}\right), n=1,2, \ldots$ Call $F^{(n)}$ the set of $n$-points of $\Gamma$. If $\phi_{i_{1}}, \ldots, \phi_{i_{n}}$ is any sequence of transformations in $\left\{\phi_{1}, \ldots, \phi_{\mu}\right\}$, call $F_{i_{1}}, \ldots, i_{n}=\phi_{i_{1}} \circ \ldots \circ \phi_{i_{n}}(F)$ an $n$-cell. Say that $\Gamma_{i_{1}, \ldots, i_{n}}=\phi_{i_{1}} \circ \cdots \circ \phi_{i_{n}}(\Gamma)$ is the associated $n$-complex.

After Lindstrøm [9], we say that $\Gamma$ is a nested fractal if it satisfies the following four conditions:

(i) Any two 1-cells $C$ and $C^{\prime}$ are connected by a sequence of 1-cells.

(ii) For $x, y \in F$, let $l_{x, y}$ be the line midway between $x$ and $y$ and let $R_{x, y}$ be reflection about $l_{x, y}$. Then $R_{x, y}$ maps $n$-cells into $n$-cells, and any $n$-cell containing points on both sides of $l_{x, y}$ is mapped into itself.

(iii) If $i_{1}, \ldots, i_{n}$ and $j_{1}, \ldots, j_{n}$ are distinct sequences, then $F_{i_{1}, \ldots, i_{n}} \neq$ $F_{j_{1}, \ldots, j_{n}}$ and $\Gamma_{i_{1}, \ldots, i_{n}} \cap \Gamma_{j_{1}, \ldots, j_{n}}=F_{i_{1}, \ldots, i_{n}} \cap F_{j_{1}, \ldots, j_{n}}$.

(iv) There exists a bounded open set $V$ such that $\phi_{1}(V), \ldots, \phi_{\mu}(V)$ are disjoint and $\bigcup_{1}^{\mu} \phi_{i}(V) \subset V$.

We recall Lindstrøm's construction of Brownian motion on a nested fractal starting from random walks on $F^{(n)}$. Treat $F^{(n)}$ as a graph by saying that $x$ and $y$ are adjacent if they belong to a common $n$-cell. Since $F^{(n)}$ is defined as the image of $F$ under a family of similitudes, we restrict our attention to $F$ momentarily.

Say that pairs of adjacent vertices $\{x, y\}$ and $\{u, v\}$ in $F$ are equivalent if $\|x-y\|=\|u-v\|$. Fix $x \in F$. Let the equivalence classes of edges of the form $\{x, u\}, u \in F$, be $C_{1}, \ldots, C_{r}$, and suppose $\left|C_{j}\right|=m_{j}, j=1, \ldots, r$. Say that a vector $\left(p_{1}, \ldots, p_{r}\right)$ is a set of basic transition probabilities if $p_{1} \geq$ $p_{2} \geq \cdots \geq p_{r} \geq 0$ and $\sum_{1}^{r} p_{j} \cdot m_{j}=1$. Let $\left(\tau_{1}, \ldots, \tau_{r}\right)$ be distributions on $(0, \infty)$ with finite second moments, and let $t_{j}=\int_{0}^{\infty} x \tau_{j}(d x), j=1, \ldots, r$. We can define a stochastic process on $F^{(n)}$ by specifying that if we arrive at 
a vertex $x$ at a time $t$, then the probability that the next vertex we visit is $y$ will be proportional to $p_{j}$ if $\{x, y\} \in C_{j}$; the distribution of the time required to move to $y$ will then be $\tau_{j}$. The evolution at time $t$ is independent of the previous evolution of the process. We will refer to such a process as a random walk with parameters $(p, \tau)$.

Let $Y_{t}^{(n)}$ be a random walk on $F^{(n)}$. For $1 \leq k \leq n$, a random walk is induced on $F^{(n-k)}$ by sampling $Y_{t}^{(n)}$ at the times it visits $F^{(n-k)}$ and using the strong Markov property. Suppose the parameters of $Y_{t}^{(n)}$ are $(p, \tau)$ and the parameters of the induced random walk on $F^{(n-k)}$ are $\left(p^{(k)}, \tau^{(k)}\right)$. In general, $p \neq p^{(k)}$ and $\tau \neq \tau^{(k)}$ for any $k$. We say that the specification $(p, \tau)$ is stable if $p=p^{(n)}$ and there exists $\lambda>0$ such that $\tau^{(n)}(d t)=\tau\left(\lambda^{n} \times d t\right)$ for $n=1,2, \ldots$.

If $\left\{X_{t}\right\}$ is a process on $\Gamma$ with continuous sample paths, let $S_{0}^{X, k}=0$ and $S_{n}^{X, k}=\inf \left\{t>S_{n-1}^{X, k}: X_{t} \in F^{(k)} \backslash\left\{X\left(S_{n-1}^{X, k}\right\}\right\}\right.$. Lindstrøm has shown the following two theorems.

Theorem 1.1. For any nested fractal $\Gamma$, there always exists at least one stable specification $(p, \tau)$.

Proof. This is Theorems V.5 and VI.5 of [9].

Theorem 1.2. Let $(p, \tau)$ be a stable specification. Then, there exists a strong Markov process $\left\{Y_{t}\right\}$ with continuous sample paths on $\Gamma$ such that for any $k \geq 0,\left\{Y\left(S_{n}^{Y, k}\right)\right\}_{n=0}^{\infty}$ is a random walk on $F^{(k)}$ specified by $\left(p, \tau\left(\lambda^{-k} \times d t\right)\right)$. Proof. This is Theorem VII.8 of [9].

Remark. Proposition IV.11 of [9] shows that it is always possible for a random walk on $F^{(1)}$ to pass directly between any arbitrary vertices of $F^{(0)}$. It follows that for any stable set of basic transition probabilities $p_{r}>0$.

Henceforward, we will always assume that we are working with some arbitrary stable specification $(p, \tau)$ and the Brownian motion $Y_{t}$ that it generates. To review, the three parameters of Brownian motion $Y_{t}$ are

$$
\begin{aligned}
& \nu=\text { the linear scaling factor }, \\
& \mu=\text { the volume scaling factor }, \\
& \lambda=\text { the time scaling factor. }
\end{aligned}
$$

We also have the following derived parameters:

$$
\begin{aligned}
d_{f} & =\log _{\nu} \mu=\text { the fractal dimension, } \\
d_{s} & =\log _{\lambda} \mu=\text { the spectral dimension, } \\
d_{w} & =\log _{\nu} \lambda=\text { the dimension of the walk. }
\end{aligned}
$$

\section{ESTIMATES OF HITTING TIMES}

We will begin with two lemmas on finite Markov chains. We first identify the stationary measure of a weighted random walk on a finite connected graph $(G, \mathscr{G})$. For each edge $\{x, y\} \in G$, let $w_{x, y}=w_{y, x}$ be a strictly positive number, and let $d_{x}=\sum_{y \in N_{x}} w_{x, y}$. Define $P_{x, y}=w_{x, y} \cdot d_{x}^{-1}$ if $\{x, y\}$ is an edge and $P_{x, y}=0$ otherwise. Then it is easy to see that $P$ is a stochastic matrix on $G \times G$. Let $X_{n}$ be a Markov chain on $G$ with transition matrix $P$. 
Lemma 2.1. For $x$ in $G$, let $m(x)=d_{x} \cdot\left(\sum_{y \in G} d_{y}\right)^{-1}$. Then, $m(x)$ is $a$ reversible stationary measure for $\left\{X_{n}\right\}$.

Proof. As $P_{x, y}=w_{x, y} \cdot d_{x}^{-1}$ we see that

$$
m(x) \cdot P_{x, y}=w_{x, y} \cdot\left(\sum_{z \in G} d_{z}\right)^{-1}=m(y) \cdot P_{y, x},
$$

so that $m$ satisfies the detailed balance equations.

Next, we wish to bound expected hitting times for a finite Markov chain. Suppose $E$ is an arbitrary finite set, and let $P$ be a stochastic matrix on $E$. Let $X_{n}$ be a Markov chain on $E$ with transition matrix $P$. Let $j \in E$ be fixed, and suppose $\theta=\inf _{i \in E} P_{i j}>0$. Let $T_{j}=\inf \left\{n: X_{n}=j\right\}$.

Lemma 2.2. $E^{i} T_{j}<(1-\theta)^{-1}$ for all $i \in E$.

Proof. Define the matrix $\bar{P}$ by

$$
\begin{array}{ll}
\bar{P}_{j j}=P_{j j} ; & \bar{P}_{i j}=\theta, \quad i \neq j ; \\
\bar{P}_{i i}=P_{i i}+P_{i j}-\theta ; & \bar{P}_{i k}=P_{i k}, \quad k \neq i, j .
\end{array}
$$

It is easy to verify that $\bar{P}$ is stochastic. Let $Z_{n}$ be a Markov chain with transition matrix $\bar{P}$. If $S_{j}=\inf \left\{n: Z_{n}=j\right\}$, then $S_{j}$ has a shifted geometric distribution with parameter $\theta$, whatever the starting state $i$. So, $E^{i} S_{j}=$ $(1-\theta)^{-1}$ for all $i$ in $E$. To compare $E^{i} T_{j}$ and $E^{i} S_{j}$, define the matrix $Q$ on $E \times E$ by

$$
\begin{aligned}
& Q((k, l),(j, j))=\theta, \\
& Q((k, l),(j, q))=\left(P_{k j}-\theta\right) \cdot(1-\theta)^{-1} \cdot \bar{P}_{l q}, \quad q \neq j, \\
& Q((k, l),(r, q))=P_{k r} \cdot(1-\theta)^{-1} \cdot \bar{P}_{l q}, \quad q, r \neq j .
\end{aligned}
$$

Straightforward calculations show

$$
\begin{aligned}
& \sum_{l, q} Q((k, l),(r, q))=P(k, r), \\
& \sum_{k, r} Q((k, l),(r, q))=\bar{P}(l, q) .
\end{aligned}
$$

It follows from this that $Q$ is a stochastic matrix that defines a coupling of $X_{n}$ and $Z_{n}$. Let $\left\{\left(X_{n}, Z_{n}\right)\right\}$ be a Markov chain on $E \times E$ starting from $(i, i)$ with transition matrix $Q$. If $i \neq j$, then if $Z_{n}=j$, either $X_{n}=j$ too, or $X_{n-1}=j$, by definition of $Q$. Thus, $T_{j} \leq S_{j}$, so that $E^{i} T_{j} \leq E^{i} S_{j}=(1-\theta)^{-1}$ for all $i$ in $E$.

We now return to Brownian motion $Y_{t}$ on $\Gamma$. For $y \in \Gamma$, let $T_{y}=$ $\inf \left\{t>0: Y_{t}=y\right\}$.

Lemma 2.3. Let $F^{*}$ be an $n$-cell in $\Gamma$ and suppose $x, y \in F^{*}$. There exists $a$ constant $B$ independent of $x, y$, and $F^{*}$ such that $E^{x} T_{y} \leq B \cdot(\mu / \lambda)^{n}$.

Remark. Corollary IX.5 of [9] shows that $\lambda>\mu$. So Lemma 2.3 shows that the expected hitting times on an $n$-cell are bounded by a decreasing geometric sequence. 
Proof. Suppose $Y_{0}=x$. Let $S_{1}=\inf \left\{t: Y_{t} \in F^{(n)} \backslash\{x\}\right\}$, and let $S_{k}=$ $\inf \left\{t: Y_{t} \in F^{(n)} \backslash\left\{Y\left(S_{k-1}\right)\right\}\right\}, k=1,3, \ldots$ Define $T_{1}=S_{1}, T_{k}=S_{k}-S_{k-1}$, $k=2,3, \ldots$. By the strong Markov property, $\left\{Y\left(S_{k}\right)\right\}$ is a weighted random walk on $F^{(n)}$. We can write

$$
E T_{y}=E\left[E\left[\sum_{1}^{N_{y}} T_{i} \mid Y\left(S_{0}\right), \ldots, Y\left(S_{N_{y}}\right)\right]\right],
$$

where $N_{y}=\inf \left\{k: Y\left(S_{k}\right)=y\right\}$. By the strong Markov property, $T_{i}$ is conditionally independent of $Y\left(S_{1}\right), Y\left(S_{2}\right), \ldots$, given $Y\left(S_{i-1}\right)$ and $Y\left(S_{i}\right)$. For an arbitrary index $i \leq N_{y}$, suppose $Y\left(S_{i-1}\right)=u$ and $Y\left(S_{i}\right)=v$, where $(u, v)$ is in the equivalence class $C_{k}$. Then the conditional distribution of $T_{i}$ will be $\tau_{k}\left(\lambda^{-n} \times d t\right)$ and $E\left[T_{i} \mid Y\left(S_{i-1}\right)=u, Y\left(S_{i}\right)=v\right]=\lambda^{-n} \cdot t_{k}$. Let $t^{*}=\max \left\{t_{1}, \ldots, t_{r}\right\}$. Then

$$
\begin{aligned}
E T_{y} & =E\left[\sum_{1}^{N_{y}} E\left[T_{i} \mid Y\left(S_{0}\right), \ldots, Y\left(S_{N_{y}}\right)\right]\right] \\
& =E\left[\sum_{1}^{N_{y}} E\left[T_{i} \mid Y\left(S_{i-1}\right), Y\left(S_{i}\right)\right]\right] \leq \lambda^{-n} t^{*} E\left[N_{y}\right],
\end{aligned}
$$

since $N_{y}$ is measurable with respect to $\sigma\left(Y\left(S_{0}\right), \ldots, Y\left(S_{N_{y}}\right)\right.$.

Define a sequence of "special" stopping times $S_{0}^{\prime}, S_{1}^{\prime}, \ldots$ by letting $S_{0}^{\prime}=0$ and taking $S_{k+1}^{\prime}=\inf \left\{S_{n}>S_{k}^{\prime}: Y\left(S_{n}\right) \in F^{*}\right.$ and $\left.Y\left(S_{n-1}\right)=Y\left(S_{k}^{\prime}\right)\right\}$. Define $M_{y}$ as the smallest $m$ such that $Y\left(S_{m}\right)=y$ and $S_{m}$ is special. Clearly $M_{y} \geq$ $N_{y}$. We can write

$$
M_{y}=\sum_{i=1}^{K}\left(\sum_{j=1}^{V_{i}} U_{i j}+1\right),
$$

where $K$ is the number of special stopping times before $S\left(M_{y}\right), V_{i}$ is the number of excursions from $Y\left(S_{i}^{\prime}\right)$ into $F^{(n)} \backslash F^{*}$ that $Y$ takes between $S_{i}^{\prime}$ and $S_{i+1}^{\prime}$, and $U_{i j}$ is the length of the $j$ th such excursion. Note that nested fractals do contain vertices belonging to a single $n$-cell. Thus, $V_{i} \equiv 0$ may occur for certain vertices; in this case, $U_{i j}$ is undefined.

We can regard $Y\left(S_{1}^{\prime}\right), Y\left(S_{2}^{\prime}\right), \ldots$ as a Markov chain on $F^{*}$, with transition probabilities given by $\left\{p_{1}, \ldots, p_{r}\right\}$. Since $\left\{p_{1}, \ldots, p_{r}\right\}$ is a stable set of basic transition probabilities, $p_{1} \geq \cdots \geq p_{r}>0$. Thus $P\left[Y\left(S_{1}\right)=u \mid Y\left(S_{2}\right)=y\right] \geq p_{r}$ for all $u$ in $F^{*}$. By Lemma 2.2,

$$
E K \leq\left(1-p_{r}\right)^{-1} \text {. }
$$

To estimate $V_{i}$, define the multiplicity of a vertex $v$ in $F^{(n)}$ as the number of $n$-cells that contain $v$. Let $\rho$ be the maximum multiplicity of a vertex in $F^{(n)}$. Then, $V_{i}$ is always dominated by a geometric random variable with parameter $\rho^{-1}$. Thus,

$$
E V_{i} \leq \rho-1 \text {. }
$$

Suppose $V_{i} \not \equiv 0$. Then $U_{i j}$ is the number of steps required by a weighted random walk $\tilde{Y}_{l}$ on $F^{(n)}$ starting from some vertex $z \neq y$ to return to $z$, given 
that $\tilde{Y}_{1} \notin F^{*}$. Necessarily, the multiplicity of $z$ is at least 2, so $P\left[\tilde{Y}_{1} \notin F^{*}\right] \geq 1 / 2$. Let $R_{z}$ be the number of steps required for $\widetilde{Y}_{l}$ to return to $z$. An elementary conditioning argument gives

$$
E^{z} R_{z} \geq E^{z}\left[R_{z} \mid \widetilde{Y}_{1} \notin F^{*}\right] P\left[\tilde{Y}_{1} \notin F^{*}\right] \geq \frac{1}{2} E^{z}\left[R_{z} \mid \widetilde{Y}_{1} \notin F^{*}\right]=\frac{1}{2} E U_{i j}
$$

To bound $E R_{z}$, use $\left(p_{1}, \ldots, p_{m}\right)$ as weights and apply Lemma 2.1 , to see

$$
\begin{aligned}
E R_{z} & \leq m(x)^{-1}=d_{x}^{-1} \cdot\left(\sum_{y \in F^{(n)}} d_{y}\right) \leq 1 \cdot 2 \cdot\left(\sum_{\text {all edges }} p_{x, y}\right) \\
& \leq 2 \cdot p_{1} \cdot\left(\# \text { of edges in } F^{(n)}\right) \leq 2 \cdot p_{1} \cdot A \cdot \mu^{n} .
\end{aligned}
$$

We condition on $K, V_{1}, \ldots, V_{K}$ and apply (2.6) and (2.7), giving

$$
\begin{aligned}
E\left[M_{y}\right] & =E\left[E\left[\sum_{i=1}^{K}\left(\sum_{j=1}^{V_{i}} U_{i j}+1\right) \mid K, V_{1}, \ldots, V_{K}\right]\right] \\
& =E\left[\sum_{i=1}^{K}\left(\sum_{j=1}^{V_{i}} E\left[U_{i j}+1 \mid K, V_{1}, \ldots, V_{K}\right]\right)\right] \\
& \leq E\left[\sum_{i=1}^{K} V_{i}\right] \cdot\left(2 \cdot 2 \cdot p_{1} \cdot A \cdot \mu^{n}+1\right) .
\end{aligned}
$$

Similarly, conditioning on $K$ and applying (2.5) and (2.4) gives

$$
E\left[\sum_{i=1}^{K} V_{i}\right] \leq E K \cdot(\rho-1) \leq \frac{\rho-1}{1-p_{r}} .
$$

Combine (2.8) and (2.9) to get

$$
E M_{y} \leq \frac{4}{1-p_{r}} \cdot\left[(\rho-1) \cdot p_{1} \cdot A \cdot \mu^{n}+1\right]
$$

Together with (2.2), this gives

$$
\begin{aligned}
E T_{y} & \leq E M_{y} \cdot \lambda^{-n} t^{*} \\
& \leq 4 t^{*} \cdot \frac{(\rho-1)}{1-p_{r}} \cdot A\left(\frac{\mu}{\lambda}\right)^{n}+(\rho-1) \cdot \lambda^{-n} \leq B\left(\frac{\mu}{\lambda}\right)^{n} .
\end{aligned}
$$

Theorem 2.4. Let $x, y \in \Gamma$ with $\|x-y\|<\nu^{-n}$. Then there exists a constant $D$ independent of $x$ and $y$ such that $E^{x} T_{y}<D(\mu / \lambda)^{n}$.

Proof. Let $F^{(\infty)}=\bigcup_{0}^{\infty} F^{(n)}$. Suppose first that $x, y \in F^{(\infty)}$ and that there is an $n$-complex $\Gamma^{*}$ such that $x, y \in \Gamma^{*}$. There exists $m \geq n$ such that $x, y \in F^{(m)}$. If $m=n$, then the theorem reduces to Lemma 2.3, and $E^{x} T_{y}<$ $B(\mu / \lambda)^{-n}$. So, suppose $m>n$, and assume that if $u, v \in F^{(m-1)}$ then $E^{u} T_{v}<$ $\sum_{n}^{m-1} 2 B(\mu / \lambda)^{k}$. There must exist $u, v \in F^{(m-1)} \cap \Gamma^{*}$ such that $\{x, u\}$ and $\{y, v\}$ belong to the same $m$-cells. Then

$$
\begin{aligned}
E^{x} T_{y} & \leq E^{x} T_{u}+E^{u} T_{v}+E^{v} T_{y} \\
& \leq B(\mu / \lambda)^{m}+\sum_{k=n}^{m-1} 2 B(\mu / \lambda)^{k}+B(\mu / \lambda)^{m} \leq \sum_{k=n}^{m} 2 B(\mu / \lambda)^{k},
\end{aligned}
$$


by the inductive hypothesis and Lemma 2.3. However,

$$
\sum_{k=n}^{m} 2 B(\mu / \lambda)^{k} \leq \sum_{k=n}^{\infty} 2 B(\mu / \lambda)^{k}=2 B \cdot(\mu / \lambda)^{n} \cdot \lambda /(\lambda-\mu) .
$$

Next, suppose $x, y \in F^{(\infty)}$, but no $n$-complex contains both $x$ and $y$. Since the diameter of an $n$-complex is at least $\nu^{-n}$, it follows that $x$ and $y$ belong to adjoining $n$-complexes, say $\Gamma_{a}$ and $\Gamma_{b}$. If we let $z \in \Gamma_{a} \cap \Gamma_{b}$, then $z \in \Gamma_{\infty}$ and $\|x-z\|$ and $\|y-z\|$ are both less than $\nu^{-n}$. Applying (2.12), we have

$$
E^{x} T_{y} \leq E^{x} T_{z}+E^{z} T_{y} \leq 4 B \cdot(\mu / \lambda)^{-n} \cdot \lambda /(\lambda-\mu) .
$$

If $x \in F^{(\infty)}$ and $y \in \Gamma \backslash F^{(\infty)}$, then there exist $n$-complexes $\Gamma_{n}, n=1,2, \ldots$, such that $y=\bigcap_{1}^{\infty} \Gamma_{n}$. If $x \in F^{(\infty)}$ and $\|x-y\|<\nu^{-n}$, then there exists $N$ such that $m \geq N$ implies $\sup \left\{\|x-z\|: z \in \Gamma_{m}\right\} \leq \nu^{-n}$. Let $T_{n}=$ $\inf \left\{t: Y_{t} \in \Gamma_{n}\right\}$. Then $T_{n} \leq T_{y}$ and $T_{n} \uparrow T_{y}$ by the sample continuity of $Y_{t}$. By monotone convergence,

$$
E^{x} T_{y}=\lim _{n \uparrow \infty} E^{x} T_{n} \leq 4 B \cdot \lambda /(\lambda-\mu) \cdot(\mu / \lambda)^{n} .
$$

Finally, let $x \in \Gamma \backslash F^{(\infty)}$ and suppose $\|x-y\|<\nu^{-n}$. For $n=1,2, \ldots$ there exists $x_{n} \in F^{(n)}$ such that $x_{n} \rightarrow x$ as $n \rightarrow \infty$. Since $T_{y}$ is a lower semicontinuous function and $Y_{t}$ is a Feller process, it follows that

$$
E^{x} T_{y} \leq \liminf _{n \rightarrow \infty} E^{x_{n}} T_{y} \leq 4 B \cdot \lambda /(\lambda-\mu) \cdot(\mu / \lambda)^{n} .
$$

Take $D=4 B \cdot \lambda /(\lambda-\mu)$. This completes the proof.

Corollary 2.5. $E^{x} T_{y} \leq C \cdot\|x-y\|^{q}$ where $q=\log _{\nu}(\lambda / \mu)$.

Proof. This follows directly from Theorem 1 , if we take $C=D \lambda / \mu$.

Remark. In terms of the dimensions introduced at the end of $\S 1, q=d_{w}-d_{f}$. This agrees with results of Barlow and Perkins [2] for the Sierpinski gasket and Krebs [7] for the Vicsek snowflake.

\section{ApPLicAtions}

In this section, we apply the bound in Theorem 2.4 to study the behavior of Brownian motion on a nested fractal. An immediate application is to show recurrence of $\left\{Y_{t}\right\}$.

Proposition 3.1. $\left\{Y_{t}\right\}$ is point recurrent.

Proof. Let $Y_{t}^{0}$ be a random walk on $F^{(0)}$ with parameters $(\lambda, \tau)$. Then it is trivial to see that $Y_{t}^{0}$ is point recurrent. Since $E^{x} T_{y}$ is bounded for $x \in F^{(0)}$, $y \in \Gamma$, it follows that $Y_{t}$ itself is point recurrent.

A second application of the hitting time bound is to show that Brownian motion on a nested fractal has a stationary distribution given by normalized Hausdorff measure on the fractal and has transitions that are absolutely continuous with respect to its stationary distribution. Since these results are quite similar to those in [7], proofs will be sketched.

Recall that $\lambda$ is the time scaling parameter for Brownian motion on $\Gamma$. For $n=1,2, \ldots$ let $\widetilde{Y}_{t}^{(n)}$ be a random walk on $F^{(n)}$, with transition distribution the same as $Y_{t}^{(n)}$, but with transition times distributed exponentially with parameter $\lambda^{n}$. 
Theorem 3.2. Let $Y_{t}$ and $Y_{t}^{\prime}$ be two independent copies of Brownian motion on $\Gamma$. Let $T_{M}=\inf \left\{u: Y_{u}=Y_{u}^{\prime}\right\}$. Then $T_{M}<\infty$ a.s.

Sketch of Proof. By Aldous [1], if $\tilde{Y}^{(n)}$ and $\tilde{Y}^{\prime(n)}$ are two independent random walks on $F^{(n)}$, and if $T^{(n)}$ is the first time that $\widetilde{Y}^{(n)}$ and $\widetilde{Y}^{(n)}$ meet, then $E T^{(n)} \leq C \cdot \max _{i, j} E^{i} T_{j}$. A calculation similar to that in Theorem 2.4 shows that $\max _{i, j} E^{i} T_{j}$ is bounded independently of $n$. Since $\tilde{Y}^{n} \rightarrow Y_{t}$ in distribution, it follows that $E T_{M} \leq \liminf E T^{(n)}<\infty$. Therefore, $T_{M}<\infty$ a.s.

Let $\eta$ be $\log _{\nu} \nu$-dimensional Hausdorff measure restricted to $\Gamma$ and normalized so that $\eta(\Gamma)=1$. Let $\eta_{t}$ be the distribution of $Y_{t}$.

Theorem 3.3. $Y_{t}$ has stationary distribution $\eta$ and $\eta_{t} \rightarrow \eta$ in total variation norm.

Sketch of Proof. $\tilde{Y}^{(n)}$ has a stationary distribution $\eta_{n}$ for each $n$ that is concentrated on $F^{(n)}$. If we regard $\left\{\eta_{n}\right\}$ as a sequence of measures on $\Gamma$, tightness allows us to choose a weakly convergent subsequence $\eta_{n}^{\prime}$. Let $\eta_{n}^{\prime} \rightarrow \eta_{0}$. It is easy to see by weak convergence that $\eta_{0}$ is a stationary measure for $Y_{t}$.

To see that $\eta_{0}$ is actually normalized Hausdorff measure, the scaling property of $Y_{t}$ shows that for any measurable set $A, \eta_{0}(A)=\eta_{0}\left(A^{(1)}\right)$. But Theorem 4.4(1) in [6] shows that this property characterizes Hausdorff measure on $\Gamma$, within a normalization.

Theorem 3.4. For all $x \in \Gamma$ and all $t>0, \eta_{t}$ is absolutely continuous with respect to $\eta$.

Sketch of Proof. Theorem 3.3, together with the scaling property of Brownian motion, shows that $\eta(A)=0$ implies $\eta_{t}(A)=0$ for all $t$.

Finally, we wish to apply the bound in Corollary 2.5 to study local times for $Y_{t}$. We will begin by showing that a local time exists at every point of $\Gamma$. Let $x \in \Gamma$, and recall $T_{x}=\inf \left\{t>0: Y_{t}=x\right\}$. We say that $x$ is regular for $\{x\}$ if $P^{x}\left[T_{x}=0\right]=1$.

Lemma 3.5. Every point $x$ of $\Gamma$ is regular for $\{x\}$.

Proof. For any $y \in \Gamma$,

$$
\begin{aligned}
P^{x}\left[T_{x}>\varepsilon\right] & \leq P^{x}\left[T_{y}>\varepsilon / 2\right]+P^{x}\left[T_{y} \leq \varepsilon / 2, T_{y}+T_{x}\left(\Theta_{T_{y}}\right)>\varepsilon\right] \\
& \leq P^{x}\left[T_{y}>\varepsilon / 2\right]+P^{y}\left[T_{x}>\varepsilon / 2\right] \leq(2 / \varepsilon) \cdot\left(2 C\|x-y\|^{q}\right)
\end{aligned}
$$

where the second inequality tacitly applies the strong Markov property. As $y$ is arbitrary, $P^{x}\left[T_{x}>\varepsilon\right]=0$. As $\varepsilon$ is also arbitrary, $P^{x}\left[T_{x}=0\right]=0$.

To apply this lemma, we cite a general theorem about Markov processes.

Theorem 3.6. A necessary and sufficient condition that there exists a local time for a Markov process $X$ at $x$ is that $x$ be regular for $\{x\}$.

Proof. This is the first part of Theorem V.3.13 of [3].

Corollary 3.7. There exists a local time for every point $x \in \Gamma$.

Besides showing that local times exist, Corollary 2.5 allows us to estimate their modulus of continuity. For $u>0$, let

$$
p(u)=\sup _{\substack{x, y \in \Gamma \\\|x-y\|<u}}\left(1-E^{x} e^{-T_{y}} \cdot E^{y} e^{-T_{x}}\right)^{1 / 2} .
$$


Now, for any $x$ and $y, E^{x} e^{-T_{y}} \geq 1-E^{x} T_{y}$, so that

$1-E^{x} e^{-T_{y}} \cdot E^{y} e^{-T_{x}} \leq 1-\left(1-E^{x} T_{y}\right)\left(1-E^{y} T_{x}\right) \leq E^{x} T_{y}+E^{y} T_{x} \leq 2 C \cdot\|x-y\|^{q}$.

Thus, $p(u) \leq C^{\prime} \cdot u^{q / 2}$. We will also need a version of Garsia's inequality for nested fractals.

Theorem 3.8. Let $F$ be a closed subset of $\mathbf{R}^{d}$ and let $\eta$ be a measure on $F$ such that there exist constants $c_{1}(F), c_{2}(F), d_{F}$ so that if $B^{F}(x, r)=F \cap\{y \in$ $\left.\mathbf{R}^{d}:|x-y| \leq r\right\}$ then

$$
c_{1}(F) r^{d_{F}} \leq \eta\left(B^{F}(x, r)\right) \leq c_{2}(F) r^{d_{F}} \quad \forall x \in F, r>0 .
$$

Let $p$ be an increasing function on $[0, \infty)$ with $p(0)=0$, and $\Psi: \mathbf{R} \rightarrow \mathbf{R}^{+}$ a nonnegative symmetric convex function, with $\lim _{u \rightarrow \infty} \Psi(u)=\infty$. Let $H$ be a compact set in $F$ and let $f: H \rightarrow \mathbf{R}$ be a measurable function. Suppose that

$$
G=\int_{H \times H} \Psi\left(\frac{|f(x)-f(y)|}{p(\|x-y\|)}\right) \eta(d x) \eta(d y)<\infty .
$$

Then there exists a constant $c_{F}$ (depending only on $c_{1}(F)$ and $d_{F}$ ) such that

$$
|f(x)-f(y)| \leq 8 \int_{0}^{\|x-y\|} \Psi^{-1}\left(\frac{c_{F} G}{u^{2 d_{F}}}\right) p(d u)
$$

for $\eta \times \eta$ almost all $x, y$ in $H$. If $f$ is continuous, then (3.2) holds everywhere. Proof. This is Lemma 6.1 of [2].

We note that if $\eta$ is normalized Hausdorff measure restricted to $\Gamma$ and $p$ is defined by (3.1), then $\eta$ and $p$ satisfy the conditions of Theorem 3.8. Take $\Psi(u)=\exp (|u|)$, and for $x \in \Gamma$, let $L_{t}^{x}$ denote the local time process at $x$. Then we have

Theorem 3.9. A version of $\left\{L_{t}^{x}\right\}$ can be chosen that is jointly continuous in $x$ and $t$. In particular, for this version,

$$
\left|L_{t}^{x}-L_{t}^{y}\right| \leq C \cdot\|y-x\|^{q / 2}[-\log \|y-x\| \wedge 0+2 / q]
$$

Proof. Let $x, y, z \in \Gamma$ be arbitrary. Let $N>0$. By Proposition 3.28 in Chapter V of [3],

$$
P^{x}\left[\sup _{0 \leq t \leq N}\left|L_{t}^{y}-L_{t}^{z}\right|>2 \delta\right] \leq 2 e^{N} e^{-\delta / p(\|y-z\|)} .
$$

As in [4] or [2], take $Y(y, z, N)=\sup _{0 \leq t \leq N}\left|L_{t}^{y}-L_{t}^{z}\right|$. Then

$$
\begin{aligned}
P^{x}\left[\exp \left(\frac{Y(y, z, N)}{4 p(\|y-z\|)}\right)>\lambda\right] & \leq P^{x}[Y(y, z, N) \geq 4 p(\|y-z\|) \log \lambda] \\
& \leq 2 e^{N} \lambda^{-2}
\end{aligned}
$$

so that

$$
E^{x} \exp \left(\frac{Y(y, z, N)}{4 p(\|y-z\|)}\right) \leq 4 e^{N} .
$$

Let $H$ be a fixed compact set in $\Gamma$. Define

$$
\begin{aligned}
\int_{H \times H} \Psi\left(\frac{\left|L_{t}^{y}-L_{t}^{z}\right|}{4 p(\|y-z\|)}\right) \eta(d y) \eta(d z) & \leq \int_{H \times H} \Psi\left(\frac{Y(y, z, N)}{4 p(\|y-z\|)}\right) \eta(d y) \eta(d z) \\
& =B_{H, N}(\omega) .
\end{aligned}
$$


Taking expectations and applying (3.3) gives $E^{x} B_{H, N} \leq 4 e^{N} \eta(H)^{2}$. By applying Theorem 3.8, we have

$$
\begin{aligned}
\left|L_{t}^{y}-L_{t}^{z}\right| & \leq 16 \int_{0}^{\|y-z\|}\left(\log B_{H, N}(\omega)-2 q \log u\right) d p(u) \\
& \leq c_{H, N}(\omega) \int_{0}^{\|y-z\|}(-\log u \vee 1) d p(u) \\
& \leq c_{H, N}(\omega)\|y-z\|^{q / 2}[-\log \|y-z\| \wedge 0+2 / q] .
\end{aligned}
$$

For any $x \in \Gamma$, let $\left\{\Gamma_{n}(x)\right\}$ be a sequence of $n$-complexes shrinking to $x$. Take

$$
\bar{L}_{t}^{x}=\limsup _{n \rightarrow \infty} \eta\left(\Gamma_{n}(x)\right)^{-1} \int_{\Gamma_{n}(x)} L_{t}^{y} \eta(d y) .
$$

As in [4], we have $\bar{L}_{t}^{x}=L_{t}^{x} \quad \eta$-a.s. and $\bar{L}_{t}^{x}$ is jointly continuous in $(x, t)$.

\section{REFERENCES}

1. D. J. Aldous, Meeting times for independent Markov chains, Technical Report No. 118, Dept. of Statistics, University of California, Berkeley, California, 1987.

2. M. T. Barlow and E. A. Perkins, Brownian motion on the Sierpinski gasket, Probab. Theory Related Fields 9 (1982), 543-623.

3. R. M. Blumenthal and R. K. Getoor, Markov processes and potential theory, Academic Press, New York, 1968.

4. R. K. Getoor and H. Kesten, Continuity of local times for Markov processes, Compositio Math. 24 (1972), 277-303.

5. S. Goldstein, Random walks and diffusions defined on fractals, Percolation Theory and the Ergodic Theory of Infinite Particle Systems (H. Kesten, ed.), 1987.

6. J. E. Hutchinson, Fractals and self similarity, Indiana Univ. Math. J. 30 (1981), 713-747.

7. W. Krebs, A diffusion defined on a fractal state space, Stochastic Process. Appl. 37 (1991), 199-212.

8. S. Kusuoka, A diffusion process on a fractal, Probabilistic Methods in Mathematical Physics (K. Ito and N. Ikeda, eds.), 1987.

9. T. Lindstrøm, Brownian motion on nested fractals, Mem. Amer. Math. Soc., vol. no. 420, Amer. Math. Soc., Providence, RI, 1990.

Department of Statistics, Florida State University, Tallahasee, Florida 32306-1037 